\title{
Effects of Electrical Leakage Currents on MEMS Reliability and Performance
}

\author{
Herbert R. Shea, Member, IEEE, Arman Gasparyan, Ho Bun Chan, Susanne Arney, Robert E. Frahm, Daniel López,
} Sungho Jin, and Robert P. McConnell

\author{
Invited Paper
}

\begin{abstract}
Electrostatically driven MEMS devices commonly operate with electric fields as high at $10^{8} \mathrm{~V} / \mathrm{m}$ applied across the dielectric between electrodes. Even with the best mechanical design, the electrical design of these devices has a large impact both on performance (e.g., speed and stability) and on reliability (e.g., corrosion and dielectric or gas breakdown). In this paper, we discuss the reliability and performance implications of leakage currents in the bulk and on the surface of the dielectric insulating the drive (or sense) electrodes from one another. Anodic oxidation of poly-silicon electrodes can occur very rapidly in samples that are not hermetically packaged. The accelerating factors are presented along with an efficient early-warning scheme. The relationship between leakage currents and the accumulation of quasistatic charge in dielectrics are discussed, along with several techniques to mitigate charging and the associated drift in electrostatically actuated or sensed MEMS devices. Two key parameters are shown to be the electrode geometry and the conductivity of the dielectric. Electrical breakdown in submicron gaps is presented as a function of packaging gas and electrode spacing. We discuss the tradeoffs involved in choosing gap geometries and dielectric properties that balance performance and reliability.
\end{abstract}

Index Terms-Anodic oxidation, charge dissipation layer, dielectric charging, MEMS reliability.

\section{INTRODUCTION}

A S MORE AND MORE MEMS devices are brought to market, it becomes increasingly important to understand potential long-term reliability issues. Unlike typical electronic devices, microelectromechanical systems (MEMS) contain moving parts, and thus MEMS reliability is often thought of in purely mechanical terms. For this reason, most studies of MEMS reliability focus primarily on mechanical aspects, as summarized in several reviews [1]-[3]. There is, however,

Manuscript received November 20, 2003; revised December 20, 2003.

H. R. Sheawas with Bell Labs, Lucent Technologies, Murray Hill, NJ 07974

USA. He is now with the Microsystems for Space Technologies Laboratory,

EPFL, CH-1015 Lausanne, Switzerland (e-mail: herbert.shea@epfl.ch).

A. Gasparyan, S. Arney, R. E. Frahm, and D. López are with Bell Labs, Lucent Technologies, Murray Hill, NJ 07974 USA.

H. B. Chan was with Bell Labs, Lucent Technologies, Murray Hill, NJ 07974 USA. He is now with the Physics Department, University of Florida, Gainesville, FL 32611 USA.

S. Jin was with Agere Systems, Murray Hill, NJ 07974 USA. He is now with the Department of Mechanical and Aerospace Engineering, University of California at San Diego, La Jolla, CA 92093 USA.

R. P. McConnell was with Lucent Technologies, Murray Hill, NJ 07974 USA.

He is now with Stanford University, Stanford, CA 94309 USA.

Digital Object Identifier 10.1109/TDMR.2004.826350 compelling evidence that electrical and electrochemical aspects are also very important. We will focus in this paper on several electrical failure modes as well as performance issues related to electrical characteristics such as leakage currents and charge traps. An emphasis will be on design for reliability: how to use one's understanding of failure modes and accelerating factors to design more robust MEMS devices [4].

Electrostatically driven MEMS devices typically consist of a conductive actuator (such as a membrane, a cantilever, a torsional plate, etc.,) suspended by micromachined springs over or next to one or more conductive electrodes. These electrodes are separated from one another and from ground by a dielectric such as $\mathrm{SiN}_{x}$ or $\mathrm{SiO}_{x}$. Applying a potential difference between the actuator and one or more of the fixed electrodes produces an attractive electrostatic force that pulls the actuator toward the electrodes. The suspension springs provide a restoring force.

MEMS devices often require high operating voltages (100-200 V) applied across small gaps (a few microns), resulting in electric fields of order $10^{8} \mathrm{~V} / \mathrm{m}$ across the dielectric. The high fields across the bulk and along the surface of the dielectric can give rise to charge injection and to leakage currents that contribute to several possible failure modes. These bulk and surface leakage currents are a strong function of voltage, temperature, and relative humidity. In this paper, we shall discuss the reliability and performance implications of leakage currents both on the surface and in the bulk of the dielectrics.

Anodic oxidation of silicon electrodes and wiring is not a novel phenomenon, but can be an important failure mode of nonhermetically packaged devices. A detailed study of the conditions under which anodic oxidation of poly-Si electrodes occur, as well as devices to provide early warning of anodic oxidation, are presented in Section II.

The positional stability of electrostatically driven MEMS devices hinges on the electrostatic charge distribution being constant in time. If the dielectrics in the device contain charge traps that can slowly fill compared with the resonant frequency of the device, then even for fixed voltages applied to the electrodes, the electric field at the actuator is time varying, and the actuator "drifts." The relationship between surface and bulk leakage currents and drift, and techniques to mitigate or eliminate drift, are presented in Section III.

Large leakage currents help to control the buildup of quasi-static charge by providing a path for charge dissipation. 
However, large leakage currents through a dielectric accelerate dielectric breakdown and lower the breakdown field. In Section IV, the breakdown of dielectrics in different packaging gases and possible shorting across small gaps due to field emission are discussed.

\section{ANODIC OXIDATION OF POLY-Si MEMS, AND STRUCTURES FOR EARLY DETECTION}

Because of its electrical and mechanical properties as well as its relative ease of processing, poly-silicon has become the material of choice in surface micromachined MEMS. The ubiquity of unpassivated poly-Si electrodes and wires in surface micromachined MEMS devices led us to investigate their reliability [5]. In dry ambients, such as the atmosphere found inside a package hermetically sealed in a dry and inert environment, poly-Si electrodes show truly impressive longevity: no signs of degradation or corrosion are observed after several months at fields close to dielectric breakdown (i.e., at fields well above those encountered during normal device operation).

Most commercial MEMS devices operate in an ultradry ambient in a hermetically sealed package so that the chip operates in a dry ambient even if the package is subjected to high relative humidity $(\mathrm{RH})$. To learn about possible failure modes in a more timely fashion, we subjected unsealed poly-Si test structures to both high relative humidities and high voltages to accelerate failures. When the test chips are operated in ambients with a $\mathrm{RH}$ of greater than $50 \%$, the most positively biased unpassivated poly-Si electrodes anodically oxidize within hours or minutes. This anodic oxidation could become a reliability concern if a MEMS device is operated in a nonhermetic package or if the hermetic package were to leak or outgas moisture.

If a poly-Si wire is allowed to fully oxidize it, becomes an open circuit, and the electrode it drives becomes nonfunctional. Partial oxidation of the electrodes can lead to a change in the capacitance between the electrode and the electrostatically actuated part, and thus to a change in the device characteristics (e.g., mirror tilt versus voltage). It is, therefore, important to not only package the chip hermetically to prevent any oxidation, but to have a means of detecting anodic oxidation in the unlikely event that the package should lose hermeticity or if significant outgassing were to occur.

We discuss here anodic oxidation of poly-silicon structures and present a poly-Si device engineered to anodically oxidize much faster than typical poly-Si-based devices. By simply measuring the resistance of the early-warning device, the onset and rate of anodic oxidation can be detected well before the MEMS device's performance is affected.

\section{A. Anodic Oxidation in Poly-Si MEMS}

If moisture is present, the adsorbed water on the surface of the dielectric between electrodes or poly-Si wiring provides a leakage path for current to flow between neighboring electrodes. We focus on the relationship between the leakage current between neighboring poly-Si electrodes and the anodic oxidation of these structures.

Several groups have observed anodic oxidation in a number of MEMS geometries [6], [7]. We have performed the first factorial study of anodic oxidation on surface micromachined poly-silicon [5] and summarize here our understanding of what parameters (relative humidity, voltage, electric field, leakage current, and electrode geometry) affect anodic oxidation. This data can be used to determine the lifetime of devices based on leak rates or outgassing rates, and to design devices more resistant to anodic oxidation.

Anodic oxidation occurs when there is a finite surface leakage current between neighboring poly-Si electrodes on the surface of the insulator in the presence of moisture. The poly-Si at the anode reacts with $\mathrm{OH}^{-}$to form $\mathrm{SiO}_{2}$ [8].

The reaction governing anodic oxidation is thought to be:

$$
\begin{aligned}
\mathrm{H}_{2} \mathrm{O} & \rightarrow \mathrm{H}^{+}+\mathrm{OH}^{-} \\
\mathrm{Si}+2 \mathrm{OH}^{-}+2 \mathrm{~h}^{+} & \rightarrow \mathrm{SiO}_{2}+\mathrm{H}_{2} \\
2 \mathrm{H}^{+}+2 \mathrm{e}^{-} & \rightarrow \mathrm{H}_{2} .
\end{aligned}
$$

As can be seen from (1), only the positively biased electrode (supplying holes, labeled $\mathrm{h}^{+}$) is oxidized, whereas the negatively biased electrode (supplying electrons, $\mathrm{e}^{-}$) is unaffected. This is exactly what we have observed in trying several different bias schemes on test structures consisting of two several-hundred-micron-long poly-Si electrodes separated by either a $2-\mu \mathrm{m}$ or a $3-\mu \mathrm{m}$ gap. The poly-Si is the Poly0 level of the Cronos MUMPS process, ${ }^{1}$ is $500-\mathrm{nm}$ thick, and is $\mathrm{n}^{+}$doped from a sacrificial phosphosilicate glass layer. The electrodes are electrically insulated from the substrate wafer by $600 \mathrm{~nm}$ of Si-rich silicon nitride. There are no moving poly-Si structures on our test chip, but the chip was released in hydrofluoric acid (HF) as are most standard surface micromachined parts.

It follows from (1) that the rate of anodic oxidation is proportional to the leakage current between electrodes on the surface of the SiN insulator [see Fig. 1(c)]. This provides a very quick way to gauge whether anodic oxidation is occurring by simply measuring the surface leakage current. Experimentally, the efficiency of this process is of the order of $2 \%$ (i.e., one $\mathrm{SiO}_{2}$ molecule is formed for every 100 electrons that flow). The total amount of oxide grown is proportional to the total charge flow (the time integral of the surface leakage current).

A combination of analytic techniques were used to verify that the observed corrosion is indeed anodic oxidation. Auger spectroscopy showed clear $\mathrm{Si}$ and $\mathrm{O}$ peaks, there was a factor of two volume expansion, the corroded anodes were soluble in HF whereas the uncorroded electrodes were not, and only the positively biased electrodes oxidized.

Relative humidity is an accelerating factor because the higher the humidity, the more water is adsorbed on the surface, and thus the larger the surface leakage current will be (there will also be more water available to supply the $\mathrm{OH}^{-}$) [9]. The surface leakage current increases roughly exponentially with relative humidity, and we find that, with all other conditions kept constant, the rate of anodic oxidation scales similarly.

Voltage is a strong accelerating factor because the leakage current is roughly proportional to the applied voltage. The electric field is also an accelerating factor (at a fixed leakage current). Much more oxidation is seen at sharp corners where the

${ }^{1}$ http://www.memsrus.com 


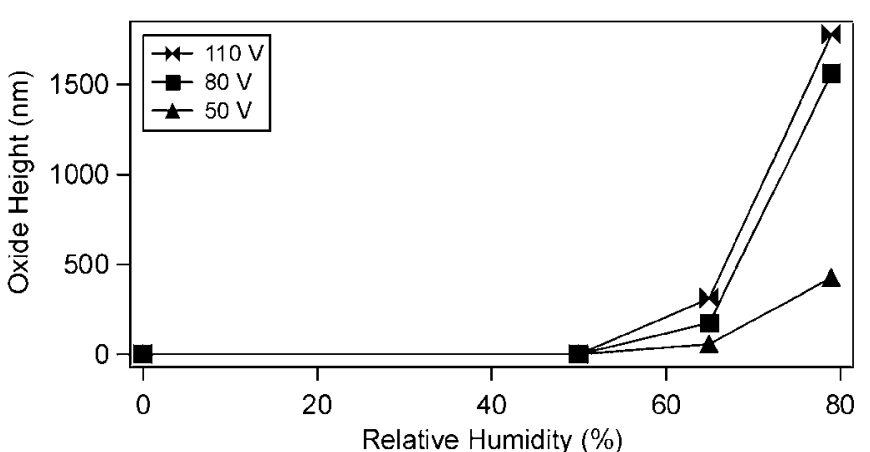

(a)

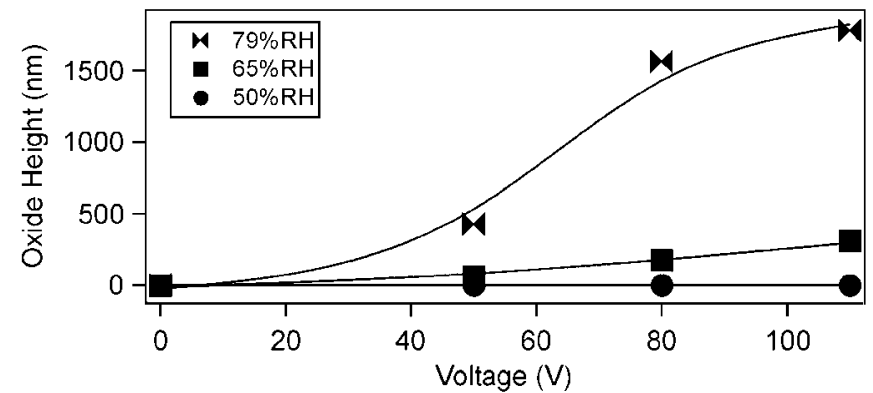

(b)

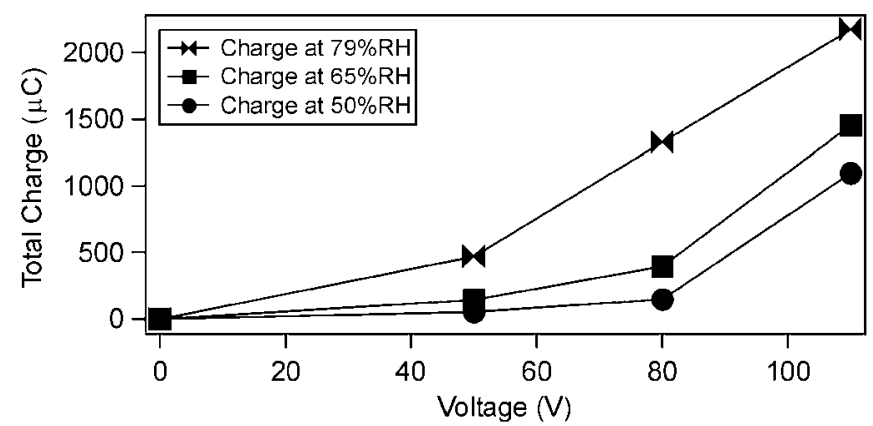

(c)

Fig. 1. Growth of anodic oxide on poly-Si electrodes after $24 \mathrm{~h}$ under various relative humidity and voltage conditions at $23^{\circ} \mathrm{C}$. (a) Oxide height versus relative humidity for the different voltages (note the threshold at $50 \% \mathrm{RH}$ ). (b) Oxide height versus voltage for the different humidities [same data as in (a)]. (c) Integrated current (i.e., total charge flow) to the oxidizing electrodes versus voltage for the different humidities. Note the correlation between (b) and (c) illustrating the proportionality between charge flow and anodic oxidation. The solid lines are merely guides to the eye.

field is concentrated. Changing the gap between electrodes or wires from 2 to $3 \mu \mathrm{m}$ has a large effect on the rate of anodic oxidation.

We did not observe any increase in anodic oxidation with increased temperature.

Fig. 1 is data taken on MUMPS-32 poly-Si (Poly 0) electrodes exposed to four levels of relative humidity at four different voltages. The amount of anodic oxide that grew on the positively biased electrodes was measured after $24 \mathrm{~h}$ and is plotted in Fig. 1(a) and (b). There appears to be a threshold in relative humidity $(\sim 50 \%)$ below which anodic oxidation does not occur. No such threshold is observed in voltage. Fig. 1(c) is a plot of the total charge that flowed to an electrode over $24 \mathrm{~h}$. Note the strong correlation between the total charge flow in Fig. 1(c) and the measured anodic oxide height in Fig. 1(b). This is consis-

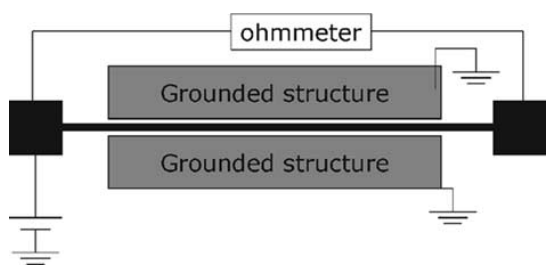

Fig. 2. Schematic drawing of a Canary wire (in black with square contact pads at both ends) with neighboring grounded structures. The wire is held at a positive bias while the wire resistance is measured with a floating ohmmeter.

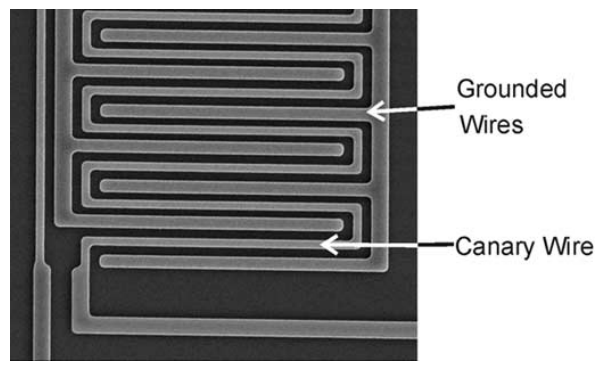

Fig. 3. SEM micrograph of a Canary device. The serpentine poly-silicon Canary wire is surrounded on both sides by grounded poly-silicon structures. The lower ends of the Canary wire are connected to bond pads (not shown). The Canary wire is fabricated from the Poly0 layer of the Cronos MUMPS process, and is $2-\mu \mathrm{m}$ wide and $0.5-\mu \mathrm{m}$ thick, with a $2-\mu \mathrm{m}$ gap between the wire and the grounded structures.

tent with (1), and allows the rate of anodic oxidation to be determined by simply measuring the surface leakage current.

\section{B. Canary Early-Warning of Anodic Oxidation}

Understanding what factors control the rate of anodic oxidation allows two goals to be reached. First, MEMS chips are designed such that anodic oxidation occurs as slowly as possible should the package leak (large gaps between electrodes, low voltage operation, passivation if possible). Second, we have designed structures that are purposefully extremely susceptible to anodic oxidation, and use them as early-warning devices. We call these devices "Canaries" in analogy to the real canaries brought down into coal mines of old: if the canaries died, the miners knew that methane levels were becoming dangerously high.

Our simple Canary devices are basically poly-Si serpentine wires placed as close possible to grounded poly-Si structures, deposited on an insulating Si-rich SiN layer. The Canary wire is biased positively with respect to ground, and its geometry is chosen such that surface leakage currents and electric fields are maximized; the wire length is maximized to enhance sensitivity. The resistance of the Canary wire is continuously monitored, as depicted schematically in Fig. 2. As the Canary anodically oxidizes, the resistance of wire increases, and this increase is readily measured. The resistance of the Canary wire provides a direct measure of the amount of wire that has been anodically oxidized. Fig. 3 is an SEM micrograph of a Canary device realized using the MUMPS process (MUMPS-36).

Fig. 4 is a plot of Canary device resistance versus time for seven devices in air with different controlled relative humidities. The higher the relative humidity, the faster the Canary oxidizes and becomes an open circuit. At $51 \%$ RH, the Canary resistance 


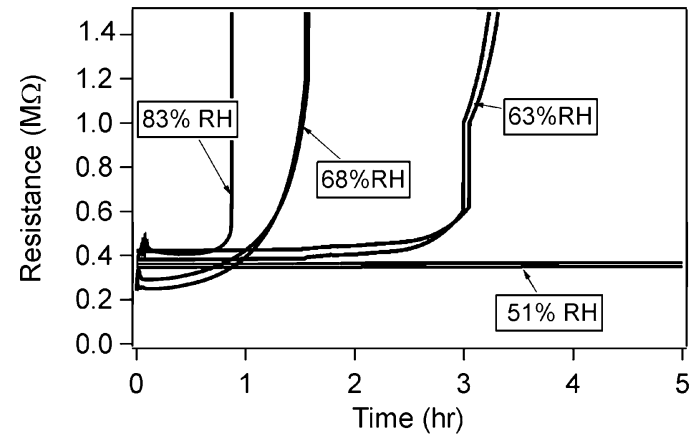

Fig. 4. Canary wire resistance versus time at $110 \mathrm{~V}$ and at $21^{\circ} \mathrm{C}$ for relative humidities of $51 \%, 63 \%, 68 \%$, and $83 \%$.

TABLE I

Time to Fully OXIDIZE A CANARY WIRE OF THE GEOMETRY SHOWN IN FIG. 3 AT 110 V FOR DIFFERENT RELATIVE HUMIDITIES

\begin{tabular}{l|c|c|c|c}
\hline$\% \mathrm{RH}$ & $51 \%$ & $63 \%$ & $68 \%$ & $83 \%$ \\
\hline $\begin{array}{l}\text { Time to open } \\
\text { circuit (hours) }\end{array}$ & $>>48 \mathrm{hr}$ & $3.6 \mathrm{hr}$ & $1.5 \mathrm{hr}$ & $0.9 \mathrm{hr}$ \\
\hline
\end{tabular}

increased by less than $0.01 \%$ over $48 \mathrm{~h}$, whereas at higher humidities the Canaries become open circuits within hours. For instance, at $68 \% \mathrm{RH}$, there is a rapid increase in the resistance of the Canary wire, which can very easily be measured with a simple ohmmeter. After $1.5 \mathrm{~h}$, the wire is fully oxidized and becomes an open circuit. The results of Fig. 4 are summarized in Table I. The total charge that flowed from the Canary wire to ground can also be used a gauge of the amount of poly-Si that has been anodically oxidized.

In order for the Canary device to provide useful early warning, the Canary must show an easily measurable increase in resistance well before the electrodes and wires on a MEMS chip degrade to a point where performance may be affected. As an example, chips developed for Lucent's LambdaRouter ${ }^{\mathrm{TM}}$ all-optical switch have been operated in 65\% RH for several weeks with no performance degradation due to anodic oxidation, whereas the resistance of Canary wires increased from approximately 0.5 to $100 \mathrm{M} \Omega$ in a few hours under similar environmental and voltage conditions. The Canary device thus offers ample early warning of a leaky or outgassing package. In fact, these devices are co-fabricated with the all chips that were shipped in commercial LambdaRouter ${ }^{\mathrm{TM}}$ all-optical switches.

Fig. 5(a) is an optical micrograph of an unused Canary wire, and Fig. 5(b) is an image of a Canary wire after $4 \mathrm{~h}$ at $60 \%$ RH and +1110 V. In Fig. 5(b), the Canary is visibly fully oxidized, whereas the grounded structures appear undamaged. The complete oxidation of a Canary wire after only $4 \mathrm{~h}$ should be contrasted with the negligible oxidation observed in wires and electrodes of a LambdaRouter ${ }^{\mathrm{TM}}$ mirror array after $24 \mathrm{~h}$ at $60 \%$ $\mathrm{RH}$ and $+110 \mathrm{~V}$. The reason is that the commercial devices were fabricated with larger gaps, lower electric fields, careful choice of polarity and electrode geometry, and much wider wires to minimize the impact of anodic oxidation (normally these devices are operated in hermetic dry packages).

Anodic oxidation can be an important failure mode for MEMS devices where electrodes and wiring is made from poly-Si or $\mathrm{Si}$. The simplest (but more expensive) solution is

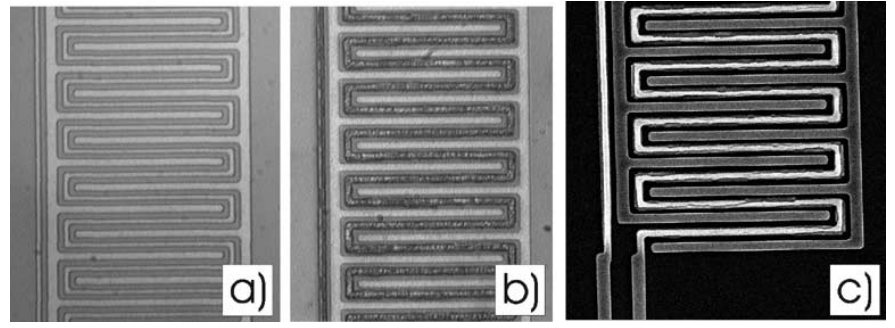

Fig. 5. (a) and (b) Optical micrographs of Canary devices. (a) Virgin Canary. (b) Canary wires after $4 \mathrm{~h}$ at $+110 \mathrm{~V}$ in $60 \% \mathrm{RH}$. Note how the Canary wire is very discolored, and has expanded due to the growth of $\mathrm{SiO}_{2}$. The Canary wire in (b) is an open circuit. (c) SEM micrograph of a fully oxidized Canary wire (bright trace). Note the cracking from the volume expansion, and how the wire only oxidizes when near grounded structures, i.e., where surface leakage currents are highest.

hermetic packaging. However, with careful engineering and good understanding of the accelerating factors (principally surface leakage currents, hence electric fields on the surface, and surface conductivity primarily due to adsorbed water), reliable MEMS devices can be fabricated even in nonhermetic packages.

\section{Dielectric Charging And Techniques FOR DRIFT MITIGATION}

Charging of the dielectrics in MEMS structures and the associated undesired time-varying electrostatic forces on actuators has been a serious performance issue for a wide range of MEMS devices including microphones, displays, micromirrors, and RF switches. Minimizing drift in MEMS devices is important so that the devices can be operated "open loop," i.e., without the need for costly and possibly bulky or power-hungry feedback electronics and sensors. Minimizing drift also greatly reduced the risk of snap-down and the associated possible stiction. Sensors such as some accelerometers and gyros that use a capacitive read-out scheme can also be very susceptible to charging, and stability of the output is greatly enhanced by the same techniques that minimize actuator drift. Designs that eliminate charging are usually radiation hard, as charges in dielectrics due to ionizing radiation will not affect device performance. There are a number of very effective techniques to eliminate or mitigate charging, but they may entail reliability tradeoffs, discussed below.

For fixed electrode voltages, the electrostatic force on a MEMS actuator is typically assumed to be constant in time. This situation, however, only holds in the ideal scenario where the dielectric contains no mobile charges or charge traps, so that all the electric fields are uniquely determined by the voltages applied to the electrodes.

When a dc bias is applied across a dielectric, charge carriers from the electrodes can be injected into various charge traps in the bulk or on the surface of the dielectric. Leakage currents can occur on the surface of the dielectric between electrodes on the dielectric held at different potentials, or through the bulk of the dielectric when there is a potential drop across the dielectric. This charge injection leads to the build up of a quasistatic charge on the surface or in the bulk of the dielectric. In addition, mobile ions (such as $\mathrm{Na}^{+}$) can migrate on the surface of 
the dielectric, and this situation is significantly worsened by the presence of any adsorbed water layers on the surface, as occurs in the presence of humidity.

The charging and discharging times to fill or empty the traps can be different by orders of magnitude, and are typically much longer than the mechanical response of the MEMS device (typically minutes or hours to charge the traps, versus the milliseconds response of the MEMS device). Unless the dielectrics are suitably electrically shielded from the actuator, the time-dependent charge on or in the dielectric gives rise to a time-dependent electrostatic force on the actuator, whose position then changes with time. This "drift" of the actuator position is of electrical, not mechanical, origin (i.e., it is not due to plastic deformation of the supporting springs).

Because of the high fields $\left(\sim 10^{8} \mathrm{~V} / \mathrm{m}\right)$ applied across dielectrics in electrostatically actuated MEMS devices, conduction is typically nonohmic, and is dominated by conduction via traps in the dielectric, and by charge injection and tunneling. The two main mechanisms are the Poole-Frenkel (PF) and Fowler-Nordheim (FN) conduction. PF conduction describes charge transport dominated by traps, and so very accurately models conduction in Si-rich SiN films commonly used in surface micromachining. FN conduction, which does not rely on defects or traps, describes tunneling of electrons from the electrode conduction band into the dielectric conduction band through part of the potential barrier at the conductor-dielectric interface. The FN model is most appropriate for conduction through silicon oxides.

The most straightforward solution to dielectric charging is to use a bipolar ac rather than dc voltage drive. This greatly reduces charging effect, but does not completely eliminate it (due to different time constant for filing traps of different polarity), and requires more complex drive electronics and significantly higher power dissipation. For these reasons, other approaches have been studied.

Much research has been done at various research labs to understand the physical origin of charging in different dielectrics, and the role of drive waveform and polarity, primarily to improve reliability and performance of capacitive RF MEMS switches (for example, see [10]-[13]). There are a number of documented ways to solve this "drift" problem. We focus here on several highly effective solutions developed at Bell Labs for use with MEMS micromirrors.

\section{A. Electrode and Dielectric Geometry}

As a concrete example, let us consider the schematic crosssection of a MEMS micromirror shown in Fig. 6. The bottom wafer ("electrode wafer") consists of a Si substrate covered by a dielectric (e.g., $\mathrm{SiO}_{x}$ ) on which electrodes are patterned (possibly with one of more wire routing layers). The small black dots represent trapped charges. A spacer is patterned on top of the electrode wafer, and an SOI wafer is flip-chipped onto the spacer. The micromirrors and supporting springs are etched out of the thin Si layer in the SOI wafer. Applying a voltage to one or more electrodes tilts the mirror. Fig. 7 is an SEM micrograph of one such mirror. The advantage of this two-chip approach for studying charging is that one has direct access to the electrodes

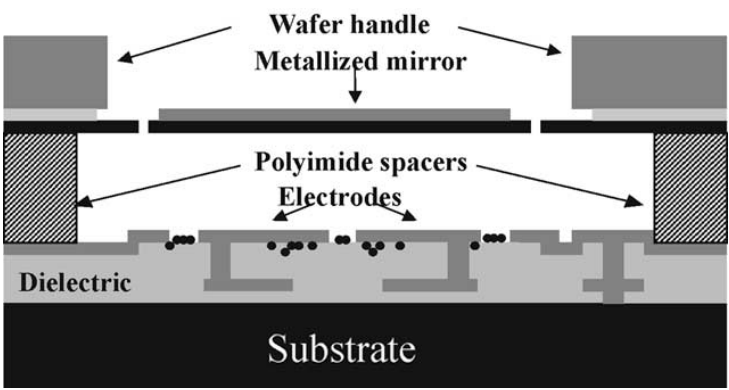

Fig. 6. Schematic cross-section of an electrostatically driven MEMS micromirror device. Mirror and electrode wafers are fabricated separately and then assembled using polyimide attachment and polyimide spacers. The black dots between and under electrodes represent trapped charge in the dielectric, as well as slowly moving mobile charges on the surface of the dielectric. The substrate is grounded while the electrodes can be grounded or held at a fixed potential.

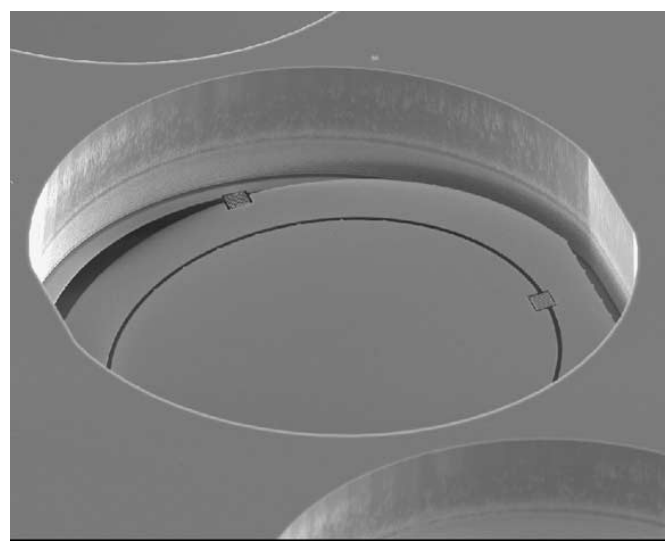

Fig. 7. SEM micrograph of a two-axis MEMS micromirror fabricated from $5 \mu \mathrm{m}$ thick single crystal silicon. The mirror diameter is $875 \mu \mathrm{m}$. The cross-section of this mirror is schematically illustrated in Fig. 6.

and dielectric prior to bonding, allowing for more anticharging techniques to be tried than for surface micromachined MEMS.

One very important and straightforward way to mitigate the effects of dielectric charging is by controlling the electrode and dielectric geometry, principally the width of the gaps with exposed dielectric between electrodes, the thickness of the electrodes, and selective etching of the dielectric. Approaches to minimize drift by changing the electrode geometry include creating overhanging electrodes to shield the actuator from the dielectric (this may present a fabrication challenge). Thicker electrodes can be effective at shielding the actuator from the charge in the dielectric, but need to be several times thicker than the electrode spacing to be effective (also a fabrication challenge).

The width of the exposed dielectric (i.e., the gap $d$ between neighboring electrodes) plays two roles. First, the more exposed dielectric there is under the actuator, the larger the electrostatic force that surface charge can exert on the actuator. This is a strong motivation for narrow gaps. Second, it is known that the dynamics of charge transport on the surface of dielectrics can be characterized using a diffusion model [14], [15]. This model suggests that to first order the saturation time $t_{s}$ scales with gap size $d$ and surface diffusion coefficient $D$ as $t_{s} \sim d^{2} / D$. For silicon oxide, $D$ is of order $10^{-11} \mathrm{~cm}^{2} / \mathrm{s}$. Narrower gaps not only reduce the area of exposed oxide thus decreasing the 


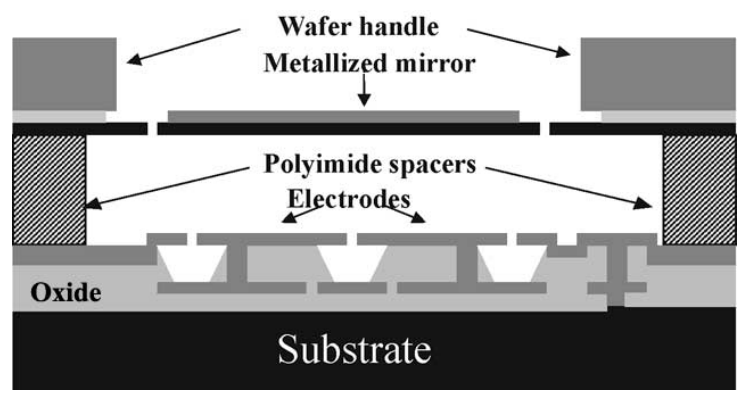

Fig. 8. Schematic cross-section of a MEMS micromirror device similar to the one shown in Fig. 6, but with the oxide selectively etched under the electrodes so that the mirror is fully electrically shielded from any trapped charge in the remaining oxide.

magnitude of charging induced drift, but also shorten the saturation time. Therefore, small gaps are essential for reducing the adverse effect of charging on mirror stability. Note, however, that minimizing anodic oxidation and increasing in-plane breakdown voltage calls for larger gaps; a careful consideration of packaging and operating conditions is required before deciding on the ideal gap size for a given application.

We have studied mirror angle drift for two MEMS micromirrors of similar geometry but with different gaps between electrodes. For a $10-\mu \mathrm{m}$ gap, we observed over 0.1 degree drift in $15 \mathrm{~h}$ (with a saturation time is of order $100 \mathrm{~h}$ ). For a $2-\mu \mathrm{m}$ gap we observed only 10 millidegrees of drift, with full saturation after $1 \mathrm{~h}$. Reducing the gap from 10 to 2 microns should to first order reduce the saturation time by 25 times, not out of line with what we observed. The acceptable micromirrors angular drift for optical crossconnect applications depends on many factors including the optical design and the nature of feedback loop. For most applications of Lucent's micromirrors, 10 millidegrees of drift is easily accommodated with no performance penalty.

We have implemented a solution based on electrode and dielectric geometry that eliminates drift in our MEMS micromirrors due to dielectric charging to below our measurement accuracy (a few millidegrees per day) [16]. Starting with an electrode design with $2-\mu \mathrm{m}$-wide gaps between electrodes, the mirror is shielded from the dielectric by undercutting the oxide in the gaps around the electrodes with a wet etch that stops on the underlying polysilicon shield layer (see Fig. 8 for a schematic). After the undercut, the mirror is exposed to only conductive surfaces, thus eliminating charging induced tilt angle drift. Charge may build up in the remaining dielectric, but because of the geometry, these charges cannot give rise to any electric field at the mirror. Fig. 9 shows an SEM micrograph of an electrode chip where the oxide has been etched away: the undercut is clearly visible. Such electrodes were used to fabricate MEMS micromirrors with drift of less than 10 millidegrees per day when held at a 5-degree tilt. We have built a $1100 \times 1100$ port optical cross-connect using these drift-free MEMS chips, and all monitored connections were observed to have better than $0.2-\mathrm{dB}$ optical loss stability over $48 \mathrm{~h}$ with no active feedback control [17].

Etching away the dielectric is a very effective solution, but care must be taken not to overetch the dielectric, which might

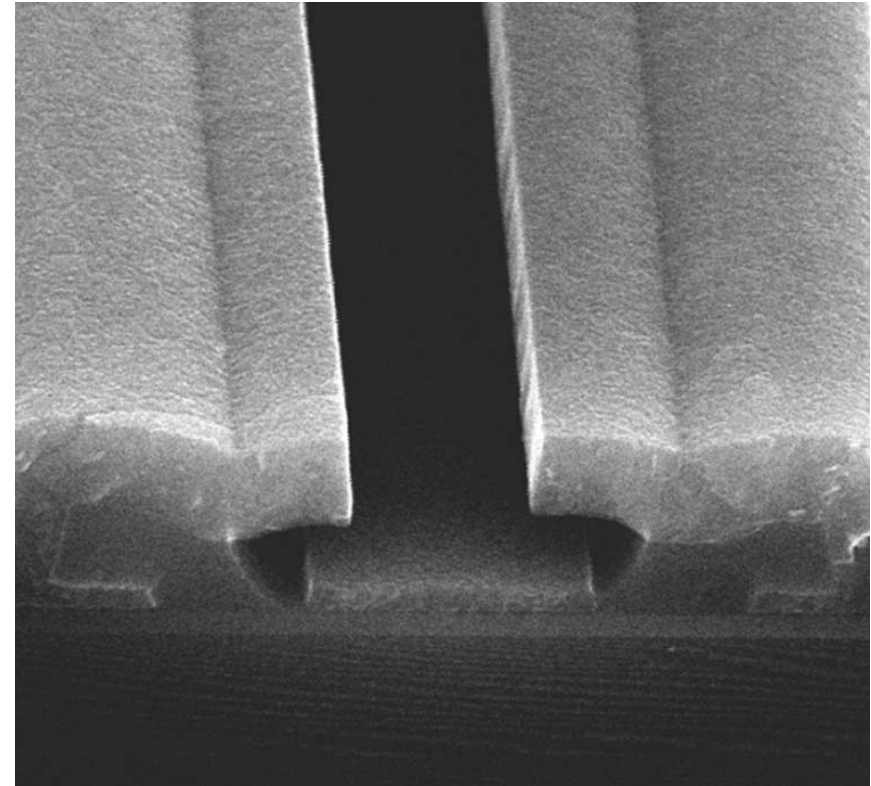

Fig. 9. SEM micrographs of the gap between two-level poly-Si electrodes, with the exposed oxide between levels removed by wet etch. The electrodes (structures on the right and left of the micrograph) are spaced by $2 \mu \mathrm{m}$. Rather than leaving oxide between the electrodes, the bottom of the gap between electrodes is covered by a grounded strip of poly-Si (running up the center of the image). The electrical potential of all surfaces is well defined, and the MEMS mirror is shielded from trapped charge in any remaining oxide. Drift in tilt angle due to dielectric charging is completely eliminated.

lead to lower breakdown voltages, and not to damage the electrodes, which typically are made from poly-silicon in order to survive the oxide etch. This solution would be very challenging to implement with $\mathrm{Al}$ electrodes because of their susceptibility to attack by HF, but is ideal for use with poly-Si electrodes when two or more levels are available.

\section{B. Tuning the Charge Dissipation Properties of the Dielectric}

There may be cases where it is not feasible to etch away the dielectric as shown in Fig. 9, for instance when building electrodes on top of CMOS circuits, or due to common limitations of the process flow, or when using a multiuser foundry process. In that case, increasing the conductivity of the dielectric is an effective means to control charge buildup in the dielectric.

A well-known solution to the charging problem is to deposit or grow a thin conductive layer on top of the dielectric in order both to bleed off surface charge and to screen bulk charge from the reflector. This charge dissipation layer (CDL) must not contain charge traps, and must be a good enough conductor to efficiently drain charge and provide electrostatic screening, while not being so conductive as to short out the electrodes by drawing too much current. The CDL typically consists of a thin film of a poor conductor such as a doped oxide. Lithium Niobate modulators have a similar charging problem to MEMS (though no moving parts). Minford and Sneh describe a CDL for $\mathrm{LiNbO}_{3}$ modulators [28]. S. Jin et al. have developed a Co-Fe-O film of tunable conductance that is an effective CDL for MEMS devices [18].

Fig. 10 is a plot of tilt angle drift for two Lucent identical micromirror devices, except that the electrodes of one device were coated with $40 \mathrm{~nm}$ of $\mathrm{CoFe}_{2} \mathrm{O}_{4}$. The Co-Fe-O CDL reduces both 


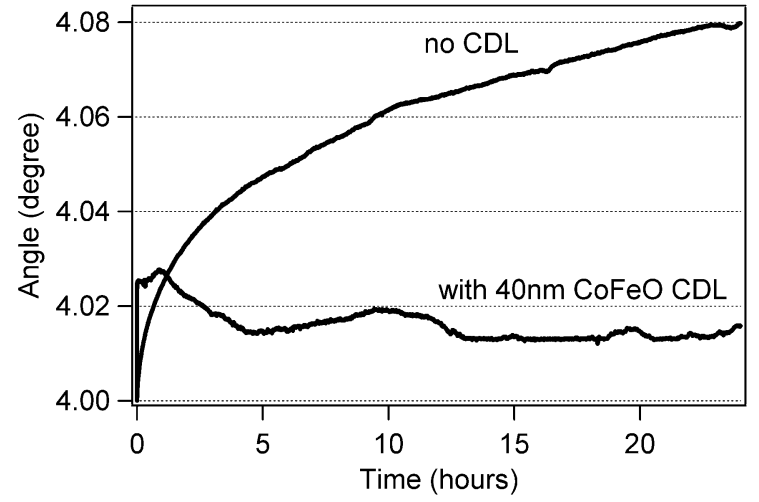

Fig. 10. Comparison of charging induced tilt angle drift for an SOI MEMS micromirror without a CDL layer on the electrodes (upper curve), and with a 40-nm-thick Co-Fe-O charge dissipating layer on the electrodes (lower curve) with approximate composition of $\mathrm{CoFe}_{2} \mathrm{O}_{4}$ and resistivity of $10^{7} \Omega-\mathrm{cm}$. Note how the CDL greatly accelerates the mirror settling and reduces drift magnitude.

the magnitude and time constant of charging related drift by a factor of more than 10 . The major advantage of Co-Fe-O is also its main potential problem: the conductivity the Co-Fe-O layer can be tuned over several orders of magnitude by annealing in oxidizing or reducing atmospheres. This allows for great flexibility in tuning of the CDL conductivity, allowing the Co-Fe-O films to be used for many different MEMS geometries and designs. The tunability however opens questions about the impact of high temperature packaging steps and about long-term stability of such coatings, which have not yet been studied.

Rather than depositing a CDL over the dielectric, the dielectric material itself can act as a CDL if its electrical transport properties are suitable: dielectric materials with larger coefficient of surface diffusion and higher bulk mobility of charge carriers are less prone to static charge buildup. However, these more "conductive" dielectrics are likely to break down at lower electrical fields. This raises an interesting reliability versus performance issue: extremely insulating dielectrics have larger breakdown fields, and thus offer higher protection against shorting through the dielectrics. Since electrostatically operated MEMS devices typically operate at voltages as high as $150 \mathrm{~V}$, this is not a negligible issue. Slightly "leaky" dielectrics can make for devices where charging is much less of an issue, but lifetime may be limited by breakdown of the dielectric. If the fabrication process flow does not allow modifications to the dielectric and electrode geometry as discussed above, the successful optimization of the dielectric layer can require a careful tradeoff between high charge mobility for elimination of charging and maintaining sufficient dielectric strength for robustness to electrical breakdown. For capacitive RF MEMS switches, Raytheon patented the approach of leaky SiN to control charging [19].

Fig. 11 is a plot of tilt angle stability for three Lucent surface-micromachined mirrors of identical geometry, each fabricated on a different wafers. Each wafer has a three slightly different compositions of the Si-rich SiN dielectric under the electrodes. Small changes to the composition of the SiN changes the film's conductivity, which was approximately $2 \times 10^{9} \Omega \mathrm{m}$, $1 \times 10^{9} \Omega \mathrm{m}$ and $5 \times 10^{8} \Omega \mathrm{m}$ for our three samples. As can clearly be seen in Fig. 11, the higher the conductivity of the SiN, the smaller the angular drift, supporting the argument above for

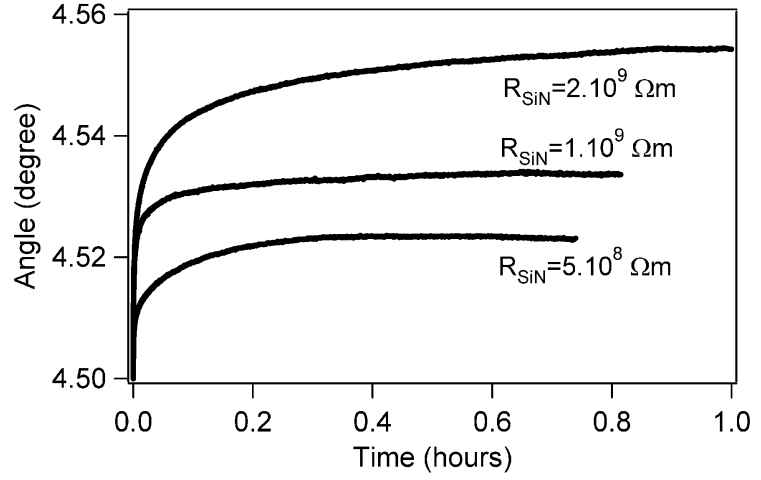

Fig. 11. Dependence of the charging induced tilt angle drift of surface micromachined mirrors on the resistivity of the silicon nitride dielectric layer: devices with lower resistivity dielectric films have less drift because charge is more readily drained away or screened. The resistivity of the silicon nitride films are approximately $2 \times 10^{9} \Omega \mathrm{m}$ (top curve), $1 \times 10^{9} \Omega \mathrm{m}$ (middle curve), and $5 \times 10^{8} \Omega \mathrm{m}$ (bottom curve).

"leaky" dielectrics for low drift (but at the expense of lowered electrical reliability).

A common model of dielectric breakdown is the "charge to failure"model [20]: the resistance of a dielectric in a large electric field remains very high even though electrons and holes are injected. These charge carriers damage the dielectric, creating more defects and charge traps. Once a critical amount of damage has been done, a conductive path is created through the dielectric, which has then broken down. So the higher the leakage current, the faster the critical charge will be reached. The thickness and conductivity of the dielectric must be carefully considered, trading off dielectric breakdown versus charge mitigation.

Rather than depositing a CDL on the electrode wafer, a thin layer of the dielectric can be made partially conductive by implanting it with metallic ions such as gold or antimony, thus creating a thin layer with a high density of metallic atoms, essentially embedding a CDL within the top surface of the dielectric. The conductivity of the implanted layer can be controlled by an appropriate choice of the dopant ion species, energy and dose, while the depth of the implanted layer is controlled by the implant energy and dopant species. The conductivity versus depth can be very accurately tuned by combining several successive implantations with different parameters (energy, dose, ion, beam angle, substrate temperature, etc.).

With ion implantation, there is typically no need for lithography or masking (one can implant metal or Si electrodes or pads without significantly degrading their electrical characteristics or ease of wire bonding); the implantation can be done at room temperature. The depth and conductivity of the CDL are readily tuned, it is possible to control film stress with the implant, and the CDL is automatically encapsulated under a thin layer of dielectric, thus protecting the CDL from corrosion or oxidation.

We studied the effectiveness of ion implantation using Si micromirrors like those shown in Figs. 6 and 7, where the electrodes and wiring consisted of $\mathrm{Al}$, and the dielectric was $\mathrm{SiO}_{2}$. The whole electrode area under the micromirrors was irradiated with either gold ions or antimony ions, thus implanting metal ions into both the $\mathrm{Al}$ electrodes and the $\mathrm{SiO}_{2}$ dielectric. The accelerating voltage for the ions was chosen such that most of 


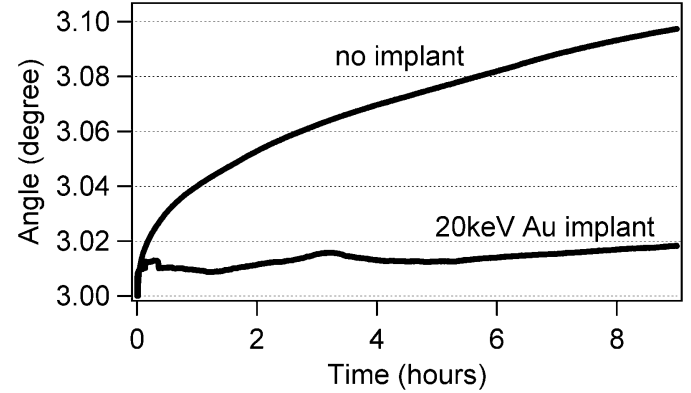

Fig. 12. Tilt angle drift data taken on a test micromirror without (top curve) and with (bottom curve) ion implantation $(20 \mathrm{keV} \mathrm{Au}$ ions with a dose of $5 \cdot 10^{16}$ ions $/ \mathrm{cm}^{2}$ ). Drift magnitude is reduced by an order of magnitude with the ion implantation.

the ions stopped within a few nanometers from the surface, creating a buried CDL that screened the mirror from trapped charge without shorting out the two electrodes. The dose of implanted ions plays an important role: if the dose is too large, too many ions accumulate which can damage the dielectric (for instance, causing cracks) or create precipitates of the implanted metal, thereby creating a short circuit between neighboring electrodes and reducing electrical reliability. If the dose is too small, the implanted ions simply damage the dielectric without creating a thin layer sufficiently conductive to dissipate or screen charge, or may create hole or electron traps, significantly worsening the charging problem.

For one geometry of Lucent micromirrors with $1-\mu \mathrm{m}$-thick $\mathrm{Al}$ electrodes on $1.5 \mu \mathrm{m}$ of $\mathrm{SiO}_{x}$ dielectric (TEOS) we found that the smallest drift of the micromirror was obtained after implantation of $20 \mathrm{keV}$ Au ions at a dose of $5 \cdot 10^{16}$ ions $/ \mathrm{cm}^{2}$. This condition corresponded to an implanted layer with $85 \%$ of the ions within a 7-nm band centered $15 \mathrm{~nm}$ below the surface. The peak concentration of $\mathrm{Au}$ ions was approximately $8 \cdot 10^{22}$ ions $/ \mathrm{cm}^{-3}$, i.e., four $\mathrm{Au}$ atoms for every $\mathrm{SiO}_{2}$ molecule. Fig. 12 is a plot of mirror tilt angle at a fixed voltage versus time without (top curve) and with (bottom curve) the ion implant condition described above. The reduction in drift amplitude is more than a factor of 10 . The optimum dose and energy, or combination of doses and energies, to effectively shield the reflector from the traps in the dielectric depends on the type of dielectric, and on the processing the film was subjected to, such as anneals, etches, etc.

There are a very wide variety of MEMS devices, designed for different purposes and made using different fabrication techniques. The choice of which drift mitigation strategy to implement depends intimately on the flexibility of the foundry or fabrication flexibility, device design, actuation technology, and on performance and reliability goals.

\section{EfFect of Electrode Gap and Packaging Gas on BREAKDOWN VOLTAGES}

The influence of the gap between electrodes has been discussed above in the context of anodic oxidation (larger gaps are more reliable) and charging (smaller gaps give higher performance). The size of the gap, the shape of the electrodes (e.g.,

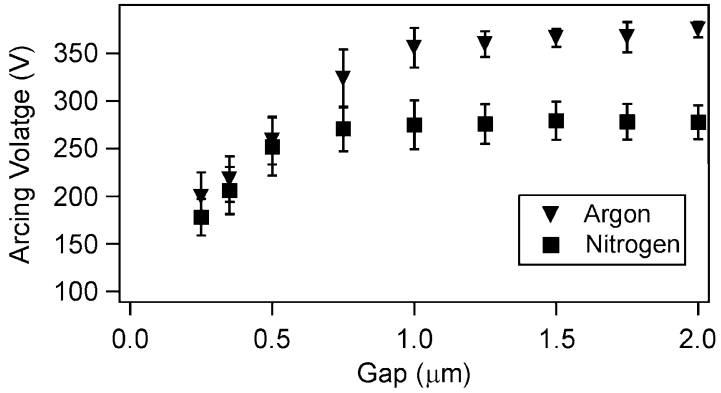

Fig. 13. Plot of breakdown voltage versus electrode gap in dry nitrogen and in argon for poly-Si electrodes. For gaps larger than $1 \mu \mathrm{m}$, a nitrogen atmosphere allows for $30 \%$ higher operating voltages.

rounded versus sharp corners), and the packaging gas (composition and pressure) all play important roles in determining the breakdown voltage.

The well-known Paschen curve [21] is often misrepresented when air gaps of the order of $5 \mu \mathrm{m}$ or less are discussed. The "standard" Paschen curve describes a gaseous breakdown model (also known as Townsend Avalanche) and predicts $360 \mathrm{~V}$ as an absolute minimum value for a breakdown in air. At 1 atmosphere, this breakdown occurs at a gap of $8 \mu \mathrm{m}$, corresponding to a breakdown electric field of $4.5 \times 10^{7} \mathrm{~V} / \mathrm{m}$. For gaps smaller and larger than $8 \mu \mathrm{m}$, the Paschen curve predicts a breakdown voltage greater than $360 \mathrm{~V}$.

However, it is very important to understand, especially as MEMS devices are scaled down, that breakdown can occur at voltages far below $360 \mathrm{~V}$ in small gaps, even if the electrode geometry is rounded to avoid field sharpening. For example, we observed a breakdown voltage of $180 \mathrm{~V}$ for gaps of $0.3 \mu \mathrm{m}$ in an argon atmosphere (see Fig. 13) and a breakdown voltage of $80 \mathrm{~V}$ has been reported for $12 \mu \mathrm{m}$ gaps in dry air at 1 atmosphere [22]. This is because at small gaps field emission becomes an important effect, and the little known "modified" Paschen curve must be used for gaps smaller than $5 \mu \mathrm{m}$ [23], [24]. There are three regimes in the modified Paschen curve. For simplicity, they are described here for dry air at 1 atmosphere. For gaps larger than 5 $\mu \mathrm{m}$, the curve looks like the regular Paschen curve with a slope of approximately $3 \mathrm{~V} / \mu \mathrm{m}$. For gaps between 2 and $5 \mu \mathrm{m}$, there is a transition region in which the breakdown voltage is almost independent of the gap. Finally, for gaps less than approximately $2 \mu \mathrm{m}$, the breakdown voltage decreases linearly to zero with the gap spacing, reflecting the increasing field emission current at smaller gaps.

The latter two regimes of the modified curve correspond well to the data shown in Fig. 13, which is a plot of breakdown voltage versus electrode gap for a series of our test electrodes. Each data point is the average of 10 measurements; the error bars are the standard deviation. There are two data sets, one for measurements made in nitrogen and in argon ambients. Note that for gaps larger than $1 \mu \mathrm{m}$, a nitrogen atmosphere allows for $30 \%$ higher operating voltages than in an argon ambient. This dependence of breakdown voltage on gas composition is well known on a macroscale [25]. Of the inert gases typically available for packaging, nitrogen provides the highest breakdown voltages.

The modified Paschen is a good guide for choosing a "safe" operating range when scaling MEMS electrodes to gaps lower 
than $5 \mu \mathrm{m}$. Care must also be taken, however, because the shape of the electrode plays a very important role. The Paschen curves describe two brass spheres. Sharp corners will concentrate the electric field and can be a point of failure. The planar nature of most MEMS electrode geometries results in a wide range of lengths scales: effectively, all gaps spacing from the air gap to the full length of the electrode are present.

Electrostatic discharge (ESD) is an important consideration and has been observed as a failure mode in MEMS [26], [27]. A static charge of several thousand volts can easily build up on a MEMS part as it is unloaded from a plastic tray, or handled by an operator who has not taken suitable precautions to ground himself. An ESD discharge can cause both electrical and mechanical damage. Possible electrical damage from the discharge includes melted wires or electrodes, pinholes or weakened dielectrics (hidden damage), and shorted transistors. Mechanical damage can result from surfaces that were not designed to come into contact under normal operating conditions colliding due to the sudden large electrostatic force, and then possible sticking (stiction) or fusing if they are at different potentials. However, because the time scale of an ESD discharge is typically nanoseconds whereas the resonant frequency of many MEMS devices is measured in kilohertz, most MEMS devices may not respond mechanically to ESD discharges. In addition to taking precautions not to expose MEMS devices to ESD discharges, the design can be changed to improve robustness. For example, mechanical stops or dimples could be added to surfaces that at first glance might not need them to minimize stiction as they should not touch in normal operation, but might after an ESD event. Another example is to passivate electrodes so as to avoid short circuits if comb fingers come into contact because of an ESD discharge.

\section{CONCLUSION}

The increasing market success of MEMS is a testament to the reliability that can be built into MEMS devices. While a poorly designed MEMS device can, of course, exhibit many failure modes, a carefully engineered and packaged MEMS device can readily meet the stringent reliability specifications of telecom and automotive applications. It is commonly perceived that the dominant failure modes of MEMS devices are mechanical, yet this is not necessarily the case, especially as design rules for actuators mature. Electrical reliability is often overlooked, which is surprising considering very high electric fields applied across the dielectrics in these MEMS devices. We have discussed in this paper the large impact both on performance (e.g., drift) and on reliability (e.g., anodic oxidation and dielectric or gas breakdown) of electrical design choices.

We have presented the relationship between leakage currents in the bulk and on the surface of the dielectrics and several performance and reliability issues. Anodic oxidation of poly-silicon electrodes can occur very rapidly in samples that are not hermetically packaged. The accelerating factors for anodic oxidation (and hence strategies to avoid it) were presented along with an efficient early warning scheme. The mechanism of charging of dielectrics and the associated "drift" of MEMS mirrors were presented, along with several very effective techniques for mitigating drift in electrostatically driven MEMS devices. Two key parameters are the gap between electrodes and the conductivity of the dielectric. For submicron gaps, the modified Paschen curve must be used, and the choice of packaging gas can be important. We provided guidance for choosing a gap and dielectric properties that balance performance and reliability, which should be very helpful for designing MEMS devices with reliability built in from the beginning, which greatly cuts time to market.

\section{ACKNOWLEDGMENT}

The authors thank the Lucent, Agere and NJNC MEMS design and fabrication teams, as well as R. Comizzoli, M. Haueis, D. Jacobson, J. Kim, R. Ruel, and C. White.

\section{REFERENCES}

[1] B. Stark, Ed., "MEMS reliability assurance guideline for space applications," Jet Propulsion Laboratory, Pasadena, CA, JPL Publication 99-1, 1999.

[2] D. M. Tanner, N. F. Smith, L. W. Irwin, W. P. Eaton, K. S. Helgesen, J. J. Clement, W. M. Miller, J. A. Walraven, K. A. Peterson, P. Tangyunyong, M. T. Dugger, and S. L. Miller, "MEMS reliability: Infrastructure, test structures, experiments, and failure modes," Sandia National Laboratories, Albuquerque, NM, Sandia Rep. SAND2000-0091. [Online.] http://mems.sandia.gov/search/micromachine/docs/000091o.pdf, 2000.

[3] W. M. van Spengen, "MEMS reliability from a failure mechanisms perspective," Microelectron. Reliabil., vol. 43, no. 7, pp. 1049-1060, 2003.

[4] S. Arney, "Designing for MEMS reliability," MRS Bull., vol. 26, no. 4, pp. 296-299, Apr. 2001.

[5] H. R. Shea, C. White, A. Gasparyan, R. B. Comizzoli, D. Abusch-Magder, and S. Arney, "Anodic oxidation and reliability of poly-Si MEMS electrodes at high voltages and in high relative humidity," in MEMS Reliability for Critical Applications, R. Lawton, Ed., 2000, vol. 4180, Proc. SPIE, pp. 117-122.

[6] G. Perregaux, S. Gonseth, P. Debergh, J.-P. Thiebaud, and H. Vuilliomenet, "Arrays of addressable high-speed optical microshutters," in Proc. 14th IEEE Int. Conf. MEMS, Jan. 2001, pp. 232-235.

[7] R. A. Plass, J. A. Walraven, D. M. Tanner, and F. W. Sexton, "Anodic oxidation-induced delamination of the SUMMiT poly 0 to silicon nitride interface," in Reliability, Testing, and Characterization of MEMS/MOEMS II, R. Ramesham and D. M. Tanner, Eds., 2003, vol. 4980, Proc. SPIE, pp. 81-86.

[8] H. J. Lewerenz, "Anodic oxides on silicon," Electrochimica Acta, vol. 37, pp. 847-864, 1992.

[9] R. B. Comizzoli, "Surface conductance on insulators in the presence of water vapor," in Materials Developments in Microelectronic Packaging: Performance and Reliability, Proc. 4th Electronic Materials and Processing Congr., 1991, p. 311.

[10] C. Goldsmith, J. Ehmke, A. Malczewski, B. Pillans, S. Eshelman, Z. Yao, J. Brank, and M. Eberly, "Lifetime characterization of capacitive RF MEMS switches," in IEEE Int. Microwave Symp. Dig., vol. 1, 2001, pp. 227-230.

[11] E. K. Chan, K. Garikipati, and R. W. Dutton, "Characterization of contact electromechanics through capacitance-voltage measurements and simulations," J. Microelectromechan. Syst., vol. 8, pp. 208-217, 1999.

[12] J. Wibbeler, G. Pfeifer, and M. Hietschold, "Parasitic charging of dielectric surfaces in capacitive microelectromechanical systems (MEMS)," Sensors Actuators A, vol. 71, pp. 74-80, 1998.

[13] J. J. Yao, "RF MEMS from a device perspective," J. Microelectromechan. Syst., vol. 10, pp. R9-R38, 2000.

[14] J. Crank, The Mathematics of Diffusion, 2nd ed. Oxford, U.K.: Clarendon, 1997, pp. 47-51.

[15] T. J. Lewis, The Movement of Electrical Charge Along Polymer Surfaces in Polymer Surfaces, D. T. Clark and W. J. Feast, Eds. New York: Wiley, 1978.

[16] A. Gasparyan, H. Shea, S. Arney, V. Aksyuk, M. E. Simon, F. Pardo, H. B. Chan, J. Kim, J. Gates, J. S. Kraus, S. Goyal, D. Carr, and R. Kleiman, "Drift-free, $1000 \mathrm{G}$ mechanical shock tolerant single-crystal silicon two-axis MEMS tilting mirrors in a $1000 \times 1000$-port optical crossconnect," in Proc. Optical Fiber Communication Conf. and Exhibit (OFC 2003), Atlanta, GA, Mar. 2003, Post-deadline paper PD36-1. 
[17] J. Kim, C. J. Nuzman, B. Kumar, D. F. Lieuwen, J. S. Kraus, A. Weiss, C P. Lichtenwalner, A. R. Papazian, R. E. Frahm, N. R. Basavanhally, D. A Ramsey, V. A. Aksyuk, F. Pardo, M. E. Simon, V. Lifton, H. B. Chan, M. Haueis, A. Gasparyan, H. R. Shea, S. Arney, C. A. Bolle, P. R. Kolodner, R. Ryf, D. T. Neilson, and J. V. Gates, "1100 × 1100 port MEMS-based optical crossconnect with 4-dB maximum loss," IEEE Photon. Technol. Lett., vol. 15, pp. 1537-1539, Nov. 2003.

[18] S. Jin, R. B. van Dover, W. Zhu, A. Gasparyan, and H. Shea, "Co-Fe-O conductive oxide layer for stabilization of actuation voltage in optical MEMS," unpublished.

[19] J. Ehmke, C. Goldsmith, Z. Yao, and S. Eshelman, "Method and apparatus for switching high frequency signals," U.S. Patent 6,391,675, May $21,2002$.

[20] M. Ohring, Reliability and Failure of Electronic Materials and Devices. New York: Academic, 1998, pp. 310-325.

[21] M. J. Madou, Fundamentals of Microfabrication: The Science of Miniaturization, 2nd ed. Cleveland, OH: CRC Press, 2002, p. 59.

[22] A. Wallash and T. Hughbanks, "Capacitive coupling effect in spark gap devices," in Proc. EOS/ESD Symp., vol. EOS-16, 1994, pp. 319-323.

[23] A. Wallash and L. Levit, "Electrical breakdown and ESD phenomen for devices with nanometer-to-micron gaps," in Reliability, Testing, and Characterization of MEMS/MOEMS II, R. Ramesham and D. M. Tanner, Eds., 2003, vol. 4980, Proc. SPIE, pp. 87-96.

[24] R. M. Schaffert, Electrophotography. New York: Wiley, 1975, pp. 514-517.

[25] M. S. Naidu and V. Kamaraju, High Voltage Engineering, 2nd ed. New York: McGraw-Hill, 1995.

[26] J. A. Walraven, J. M. Soden, D. M. Tanner, P. Tangyunyong, E. I. Cole, R R. Anderson, and L. W. Irwin, "Electrostatic discharge/electrical overstress susceptibility in MEMS: A new failure mode," in MEMS Reliability for Critical Applications, R. A. Lawton, Ed., 2000, vol. 4180, Proc. SPIE, pp. 30-39.

[27] T. Ono, Y. S. Dong, and M. Esashi, "Imaging of micro-discharge in a micro-gap of electrostatic actuator," in Proc. 13th Annu. Int. Conf. Micro Electro Mechanical Systems (MEMS 2000), Jan. 2000, pp. 651-656.

[28] J. W. Minford and O. Sneh, "Apparatus and method for dissipating charge from lithium niobate devices," U.S. Patent 5,949,944, Oct. 2, 1997.
Herbert R. Shea, photograph and biography not available at the time of publication.

Arman Gasparyan, photograph and biography not available at the time of publication.

Ho Bun Chan, photograph and biography not available at the time of publication.

Susanne Arney, photograph and biography not available at the time of publication.

Robert E. Frahm, photograph and biography not available at the time of publication.

Daniel López, photograph and biography not available at the time of publication.

Sungho Jin, photograph and biography not available at the time of publication.

Robert P. McConnell, photograph and biography not available at the time of publication. 